Bangl. J. Vet. Med. (2007). 5 (1 \& 2): 93-98

\title{
EVALUATION OF PROTECTIVE IMMUNITY AGAINST EXPERIMENTAL DIROFILARIA IMMITIS INFECTION IN BEAGLE DOGS
}

\author{
M. H. Talukder, K. Ueda, T. Hajime, S. Kanako, S. Kawamura and Y. Hikasa \\ Laboratory of Veterinary Internal Medicine, Department of Veterinary Clinical Science, Faculty of Agriculture, \\ Tottori University 680-8553, Japan
}

\begin{abstract}
Prevention of heartworm is a key goal of pet wellness. An experimental trial was carried out to induce the immunologic protection against Dirofilaria immitis infection in dogs during the period from July 2005 to November 2006. To evaluate the three protocols of immunization, dogs were separately immunized with gamma-irradiated infective larvae; with chemical abbreviation of infection; with chemical abbreviation plus Freund's complete adjuvant respectively. Each trial consisted of immunized and control groups and each group composed of two dogs. All dogs used for this study were subcutaneously challenged with 100 intact third-stage larvae at designated times after the last immunization following the above three protocols. The dogs were euthanized and necropsized between 120 to 175 days after challenge infection for the worms in the right ventricle of the heart and pulmonary arteries. Number of worms and sexes were determined. A mean of 36 worms from the immunized groups with irradiated L3; 33 worms from the chemically-abbreviated group whereas 13 worms from the chemical abbreviation plus Freund's complete adjuvant group were recovered. The percentages of the average protection in three groups were $45.80 \%, 55.05 \%$ and $77.90 \%$ respectively. The adjuvant enhanced the protective immunity against L3 challenge infection. The ELISA values do not explain the intensity of the protection, but shown adequately the immunized dogs responding to the immunization performed in this experiment.
\end{abstract}

Key words: Heartworm, protective immunity, chemical-abbreviation, gamma-irradiated larvae

\section{INTRODUCTION}

Dirofilariosis is one of the most important parasitic diseases in dogs in Japan with a high prevalence of $50 \%$ in Tokyo and 41\% in Osaka area (Roncalli et al., 1998). Heartworm infection is quite debilitating, even deadly. Furthermore, treatment is costly and difficult. It has been reported that $30.4-68.0 \%$ of worms were recovered from the lung and the heart of experimentally infected dogs (Ohishi et al., 1987). However, there is no report stating that about $100 \%$ worms have been recovered; namely, half of the inoculated larvae failed to migrate into the lung or heart, and died. The decreased viability of the larvae during harvesting might be one of the important reasons for the deaths. However, it is difficult to explain the mechanism that most researchers reported recovery rates of 30.4 to $68.0 \%$ in normal animals. There is a possibility that a part of third-stage larvae that could not reach lung or heart were eliminated by the host immune mechanism. It is reported that macaques treated with an immunosuppressant were susceptible to the infection with Dirofilaria immitis (Wong, 1974). It takes 120 days tentatively for the larvae to invade the heart and lung; hence there is a sufficient time for dogs to raise an immune response against dirofilariosis (Kotani and Powers, 1982; Kume and Itagaki, 1955). The host receives an adequate stimulus from the worms during this period and becomes responsive to the antigens of the worms. Dogs produce antibodies to canine heartworm antigens around 112 days after infection with L3 (Grieve et al., 1981). Normally there is a sufficient antibody titer against the antigen during heartworm infection but the naive dogs are not capable to resist the infection. This escape mechanism of parasites has grounded on a mimicry theory. The parasites will be eliminated if the hosts potentiate their immune response and the parasites reduce its surface antigens. The use of chemical abbreviation of immunizing infections is based on the fact that molting larvae produce functional antigens. On the basis of this background, studies on immunization of dogs against the antigen in heartworm disease were carried out. 
It has been reported that immunization with 20-Krad-attenuated L3 resulted in the resistance to the infection with D. immitis. (Media and Carlow, 1994; Wong et al., 1974). Additionally, immunization with a chemicallyabbreviated infection has been reported to make the ferrets and the dogs protected against the challenge infection with L3 (Blair and Campbell, 1981; Grieve et al., 1988). These results suggest the possibility that canine heartworm infection is protected by vaccination. The immunization described by previous authors (Grieve et al., 1988; Wong et al., 1974) was evaluated with modifications and, in addition, the enhanced efficacy of Freund's complete adjuvant was also confirmed in dogs with chemically abbreviated infection in this study.

\section{MATERIALS AND METHODS}

The experimental works were performed at the laboratory of Veterinary Internal Medicine and Veterinary Teaching Hospital of Tottori University, Japan during July 2005 to November 2006.

\section{Animals}

Twelve healthy male beagle dogs, aged 6.2 \pm 2.7 (mean \pm SD) months and weighing $8.44 \pm 2.01 \mathrm{~kg}$ (mean \pm SD) were used for this experiment. Prior to receiving in our laboratory from Fujisawa Pharma, Japan, all were bred mosquito-proof indoor and vaccinated against distemper virus, adenovirus type II, and parvovirus. During the study, they were kept in mosquito-proof individual cages in a controlled environment in $12 \mathrm{~h} \mathrm{light/dark}$ cycle. All dogs were fed standard dry food. On the basis of the results of physiological, hematologic, and serum biochemical examinations, the dogs were found healthy. The study protocol was approved by the Animal Research Committee of Tottori University. This study was designed with three separate trials of immunization protocols; Trial I (immunization with infective larvae attenuated by irradiation with ${ }^{60} \mathrm{Co}$ at a dose of 20 Krads), Trial II (with ivermectin-abbrevtion of infection), and Trial III (ivermectin abbreviation of infection plus Freund's complete adjuvant). In two trials of ivermectin-abberiation, control dogs received ivermectin treatment alone. Each trial consisted of immunized and control groups and each group composed of two dogs.

\section{Microfilariae}

Three microfilaremic dogs, naturally infected with $D$. immitis, were employed as microfilaria donors to mosquitoes, Aedes togoi. Mosquitoes were maintained and obtained from Department of Internal Medicine, Faculty of Medicine, Tottori University. The mosquitoes were fed directly canine blood for 30 min from a microfilaremic dog anesthetized with sodium pentobarbital (Meiji Kaisha, Tokyo, Japan). Infective larvae were recovered by Baermann's method from mosquitoes two weeks after blood meal. The mosquitoes were anesthetized with ether vapor and collected on gauze. The packed mosquitoes were gently crashed with a roller on a table, placed on a funnel filled up with Ringer's solution, and incubated for $1 \mathrm{~h}$ at room temperature. Larvae migrating into outer of the solution were collected in a fresh Ringer's solution and washed twice with Waymouth medium (GIBCO, Grand Island, NY, and USA) to remove the crashed component of the mosquitoes. For examining the effect of the attenuated larvae on the protection against the infection with $D$. immitis, the larvae were irradiated with ${ }^{60} \mathrm{Co}$ at a dose rate of 20 Krads in Waymouth medium. Dogs were subcutaneously infected with various numbers of the larvae in Waymouth. A $5 \mathrm{ml}$ syringe attached with an 18-gauze needle used for the injection.

\section{Immunization and challenge infection}

Groups of three experimental trials were immunized and challenged with the following schedules.

Trial I: Immunization with attenuated L3 - Two dogs were immunized three times each with a 500 attenuated L3 at intervals of 55 and 70 days. Challenge infection of four dogs with 100 intact L3 was performed 60 days after the last immunization.

Trial II: Immunization with chemical abbreviation of infection - Two dogs were infected with 500 intact L3 and then given ivermectin (CARDOMEC, Merck, Sharp and Dome, NJ, U.S.A) at an oral dose of $50 \mu \mathrm{g} / \mathrm{kg}, 60$ days after infection. Two control dogs were also treated with this drug at the same dose and time. At an interval of 35 days, this schedule was repeated three times. Challenge infection was given to four dogs with intact L3 at 30 days after the last ivermectin administration. 
Trial III: Immunization with chemical abbreviation plus Freund's complete adjuvant (FCA) - Two dogs were infected with 500 intact L3, and simultaneously injected at another site with an emulsion of FCA (FCA, DIFCO Laboratories, Detroit, MI, USA). FCA was mixed with the same volume of saline, sonicated at a maximal power and the emulsion was suspended in saline containing $2 \%$ Tween 80 with a sonicator. Finally, the adjuvant formed water-in-oil in water. The preparation in $0.8 \mathrm{ml}$, equivalent to $0.3 \mathrm{ml}$ of FCA, was injected into the back skin of the immunized dogs in eight divided doses. At an interval of 40 days, this regimen was repeated twice. After 15 days of the second regimen, all four dogs were medicated with ivermectin at an oral dose of $60 \mu \mathrm{g} / \mathrm{kg}$, and then challenge infection was accomplished in the same way as above. To confirm the humoral immune response in all immunized dogs, serum antibody was titrated prior to 7 days of challenge infection by antibodycapture EISA.

\section{Necropsy and recovery of worms}

To count the worms migrating into the heart and adjacent pulmonary arteries, all animals were euthanized with an intravenous administration of sodium pentobarbital at a dose of $60 \mathrm{mg} / \mathrm{kg}$. Immediately after euthanasia, they were necropsized and the right ventricle of the heart and lung were opened and examined. Recovered worms were kept in saline until counting and sex determination. Effectiveness of the immunization was calculated with the following formula:

$\%$ protection $=\{$ mean recovery from control dogs $\}-\{$ worm recovery from the immunized dogs $\} /$ mean worm recovery from control dogs.

\section{Antigens for ELISA}

Adult worms were collected from naturally infected dogs and stored at $-40^{\circ} \mathrm{C}$ until processing. For the antigen preparation the worms were lyophilized and crashed with a mortar and pestle. The minute fragments of the worms were dehydrated in $0.01 \mathrm{M}$ phosphate buffered saline and sonicated on ice until the cuticle was separated from the inner structures. The preparation was washed with PBS, centrifuged for $1 \mathrm{hr}$ at $10,000 \mathrm{x} \mathrm{g}$ until the protein in the supernatant disappeared. The sediments were resuspended in $0.05 \mathrm{M}$ Tris-HCL buffer containing $1 \%$ SDS and 5\% beta-mercaptoethanol (Sigma Chemicals) and sonicated for $15 \mathrm{~min}$ on ice. The homogenate was extrated overnight at $4^{0} \mathrm{C}$ and centrifuged for $1 \mathrm{hr}$ at $10,000 \mathrm{x}$ g at $4^{0} \mathrm{C}$. This supernatant was dialyzed against $0.01 \mathrm{M}$ PBS for three days and centrifuged for $1 \mathrm{hr}$ at $10,000 \mathrm{x}$ g at $4^{0} \mathrm{C}$ to remove the precipitate. The supernatant was used for ELISA after determining protein concentration in the supernatant with Bradford's method.

\section{Enzyme Linked Immunosorbent Assay}

Antibody-capture ELISA was performed with immunized canine sera. Wells of a micro plate (Wako Pure Chemical Industries, Ltd, Osaka, Japan) were coated with D. immitis antigen in 0.1M carbonate buffer (pH 9.6). Sera diluted 1:100 were placed in the wells and incubated for $1 \mathrm{hr}$ at $37^{\circ} \mathrm{C}$. After three washes, appropriately diluted anti-canine IgG antibody conjugated with peroxidase was added to the wells and incubated again in the same way as above. After free conjugated antibody was washed away, 2, 2'-azino-bis (Wako Pure Chemical Industries, Ltd, Osaka, Japan) at a concentration of $0.3 \mathrm{mg} / \mathrm{ml}$ and $0.01 \%$ peroxide as a substrate were added to the wells. The plate was placed for about $20 \mathrm{~min}$ at room temperature and $1 \%$ sodium dodecyl sulfate (SDS, Wako Pure Chemical Industries, Ltd, Osaka, Japan) solution was added to the wells to stop the reaction. The absorbance was read with a microplate reader. ELISA values were calculated with the following formula:

ELISA value $=($ sample absorbance - absorbance of the negative control) $/$ (the absorbance of the positive control) X 100.

\section{RESULTS AND DISCUSSION}

From the two immunized dogs of trial I - immunization with attenuated L3; 35 and 37 worms were recovered 150 days after the challenge infection. These dogs showed 44.3 to $47.3 \%$ protections. Details are shown in Table 1. From the two dogs immunized with the chemical abbreviation of the infection, 30 and 36 worms were found 175 days after challenge infection. These dogs revealed 59.1 to $51.0 \%$ protections (Table 1 ). Dogs immunized with chemical abbreviation of the infection stimulated with FCA, recovered 15 and 11 worms, 120 days after the challenge infection. These dogs apparently 74.5 and $81.3 \%$ protected against the challenge infection (Table 1). ELISA values prior to the immunization and the challenge infection in the immunized dogs, are shown in Table 2. 
Table 1. Adult D. immitis recovered from immunized and control dogs after necropsy and percentages of protection

\begin{tabular}{|c|c|c|c|c|c|c|c|}
\hline \multirow[t]{2}{*}{ Trials } & \multirow[t]{2}{*}{ Groups } & \multirow[t]{2}{*}{ Dog ID } & \multicolumn{5}{|c|}{ Number of adult worms collected at necropsy } \\
\hline & & & Male & Female & Unidentified & Total & \% protection \\
\hline \multirow[t]{4}{*}{ Trial I } & \multirow[t]{2}{*}{ Immunized } & $\mathrm{A}$ & 15 & 20 & - & 35 & 47.3 \\
\hline & & B & 11 & 26 & - & 37 & 44.3 \\
\hline & \multirow[t]{2}{*}{ Control } & $\mathrm{C}$ & 24 & 42 & - & 66 & \\
\hline & & $\mathrm{D}$ & 30 & 37 & - & 67 & \\
\hline \multirow[t]{4}{*}{ Trial II } & \multirow[t]{2}{*}{ Immunized } & $\mathrm{E}$ & 12 & 18 & - & 30 & 59.1 \\
\hline & & $\mathrm{F}$ & 13 & 23 & - & 36 & 51.0 \\
\hline & \multirow[t]{2}{*}{ Control } & $\mathrm{G}$ & 27 & 39 & 12 & 78 & \\
\hline & & $\mathrm{H}$ & 23 & 41 & 5 & 69 & \\
\hline \multirow[t]{4}{*}{ Trial III } & \multirow[t]{2}{*}{ Immunized } & I & 6 & 9 & - & 15 & 74.5 \\
\hline & & $\mathrm{J}$ & 4 & 7 & - & 11 & 81.3 \\
\hline & \multirow[t]{2}{*}{ Control } & $\mathrm{K}$ & 22 & 30 & 6 & 58 & \\
\hline & & $\mathrm{L}$ & 26 & 32 & 2 & 60 & \\
\hline
\end{tabular}

$\%$ Protection $=\{$ Mean worm recovery from control dogs - worm recovery from immunized dogs $\} /$ Mean worm recovery from control dogs.

Table 2. Humoral immune responses of the immunized dogs

\begin{tabular}{|l|l|l|l|}
\hline \multirow{2}{*}{ Group } & ID of immunized dog & ELISA Value & \\
\cline { 3 - 4 } & & Prior to immunization & Prior to challenge infection \\
\hline \multirow{2}{*}{$\begin{array}{l}\text { Immunized with } \\
\text { infective L3 larvae }\end{array}$} & $\mathrm{A}$ & -0.59 & 80.3 \\
\cline { 2 - 4 } $\begin{array}{l}\text { Immunized with IVM } \\
\text { abbreviation }\end{array}$ & $\mathrm{B}$ & -0.06 & 89.9 \\
\cline { 2 - 4 } $\begin{array}{l}\text { Immunized with IVM } \\
\text { abbreviation plus FCA }\end{array}$ & $\mathrm{F}$ & -0.90 & 99.5 \\
\cline { 2 - 4 } & $\mathrm{J}$ & -0.71 & 31.1 \\
\hline
\end{tabular}

All dogs were immunized with 500 infective L3.

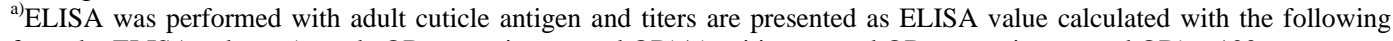
formula: ELISA value = $($ sample OD - negative control OD $) /($ positive control OD - negative control OD) $\times 100$.

Before the first immunization, ELISA values of five dogs in the immunized groups were lower than the negative control level. Only dog I had a value of 1.88. A few days before the challenge infection, their values were between 31.1 and 99.5. A lower ELISA value was found in dog F compared with others. We have focused on the mechanisms of the protection from L3 challenge infection in immunized dogs. On the basis of the description of earlier two reports (Wong et al., 1974; Grieve et al., 1988), this experiment was carried out with modifications in trial I and II. From this trial experiment, we revealed the average protection $45.80 \%$ and $55.05 \%$ in trial I immunized with gamma attenuated L3, and trial II with ivermectin-abbreviation respectively. Whereas previous two reports (Wong et al., 1974; Grieve et al., 1988) showed 100\% protection against L3 of D. immitis in dogs that greatly varied with our findings. In addition, the dogs immunized with gamma-attenuated L3 perfectly resisted the challenge infection with intact L3 only approximately 90 days after the latest immunization, but at 57 or 120 days post-immunization, the protection was reduced (Wong et al., 1974). This suggests that there is an optimal period from immunization to infection and the period is very short. However, Grieve et al. (1988) has reported that dogs immunized with the abbreviation of the L3 infection were completely protected against double challenge infections in 156 and 212 days after the last administration with ivermectin except one of four dogs. The duration from the last killing to the challenge was obviously longer than the optimal period described by Wong et al. (1974). Thus the period seemed to be not so important. 
A total of 1,500 L3 worms were used for the immunization in each one dog of trial I and II. This number of worms should be enough to develop immunity in dogs, since high antibody titers were demonstrated by ELISA in immunized dog sera before the challenge infection in this study. Additionally, dog $\mathrm{F}$ showed a relatively lower titer in the serum compared with others at this time, but there was no difference in the recovery rate of the worms among these immunized animals. The ELISA values do not explain the intensity of the protection, but shown adequately the immunized dogs responding to the immunization performed in this experiment. The recovery rates in control dogs must be reflected by viability of infective larvae used for the challenge infection. The recovery rates in nonimmunized dogs were $22-54 \%$ and $31-40 \%$ described by Wong et al. (1974) and Grieve et al. (1988) respectively. These worm recovery rates were distinct than those revealed in this study. During the experimental infection, usually we expelled immotile larvae, but were not able to separate mildly damaged worms from those used for the challenge infection. The cryptic damage of the larvae may affect the molt coming soon after injection and their survival in immunized dogs, and the damaged worms must be easily eliminated during the migration without regard to the intensity of immunity of the dogs. Thus the low viability of the larvae may produce higher percentage of the protection than the actual. On the other hand, adult worm recovery from immunized dogs with gamma-attenuated L3 fluctuated between 34\% and 9\%. (Media and Carlow, 1994). The wide fluctuation indicates that the dogs did not uniformly respond to the challenge in accordance with the immunization protocol. Because, out-bred beagles were used for this experiment, and there was no information of genetic backgrounds of dogs related to immune response. These immunization protocols might have a problem in the reproducibility of inducing the protective immunity. The experiment in trial -I and II, took 215 and 450 days from the first immunizations to necropsies, respectively.

Finally, we considered an immunologic enhancement to shorten the period of experiment and to improve the protection. To further stimulate the immune system with the excretory and secretory antigens, molt-related antigens, and dead worms, FCA was simultaneously administered with L3 injection for immunization in trial III (Table 1). Reduced recovery of the adult worm was observed by adding FCA administration to the trial-III. It has been reported that microfilariae of $D$. immitis were eliminated from the peripheral blood with antibody against their surfaces in occult dogs (Wong and Suter, 1979). Conversely, in L3 infection, antibody against $D$. immitis may not play an important role in protection of dogs. Because, even though high antibody titer was kept in peripheral blood during the tissue migration of D. immitis in its host (Yamagata et al., 1992), almost 50\% of the worms have been recovered from the heart and the pulmonary arteries in experimental infection. However, it is difficult to explain the mechanism of the protection and thus merits further study.

\section{ACKNOWLEDGEMENTS}

The authors would like to thank Fujisawa Pharma for supply of beagle dogs, and Department of Internal Medicine, Faculty of Medicine, Tottori University for the supply of parasites and also technical assistance.

\section{REFERENCES}

1. Blair LS and Campbell WC (1981). Immunization of ferrets against Dirofilaria immitis by means of chemically abbreviated infections. Parasite Immunology 3: 143-147.

2. Grieve RB, Abraham D, Grieve MM and Seibert BP (1988). Induction of protective immunity in dogs to infection with Dirofilaria immitis using chemically abbreviated infections. American Journal of Tropical Medicine and Hygiene 39: 373-379.

3. Grieve RB, Johnson MM, Jacobson RH and Cypess RH (1981). Enzyme-linked immunosorbent assay for measurement of antibody responses to Dirofilaria immitis in experimentally infected dogs. American Journal of Veterinary Research 42: 66-69.

4. Kotani T and Powers KG (1982). Developmental stages of Dirofilaria immitis in the dog. American Journal of Veterinary Research 43: 2199-2206.

5. Kume S and Itagaki S (1955). On the life-cycle of Dirofilaria immitis in the dog as the final host. British Veterinary Journal 111: 16-24.

6. Media JS and Carlow CKS (1994). An analysis of the humoral immune response of dogs following vaccination with irradiated infective larvae of Dirofilaria immitis. Parasite Immunology 16: 157-164.

7. Ohishi I, Katae H, Hayasaki M, Nakagaki K, and Tada Y (1987). Prophylactic activity of ivermectin against Dirofilaria immitis infection in dogs: Establishment of infective dose and administration schedule. Japanese Journal of Veterinary Science 49: 439-445. 
8. Roncalli RA, Yamane Y and Nagata T (1998). Prevalence of Dirofilaria immitis in cats in Japan. Veterinary Parasitology 75: 81-89.

9. Wong MM (1974). Experimental dirofilariosis in macaques: Susceptibility and host responses to Dirofilaria immitis, the dog heartworm. Transaction of Royal Society for Tropical Medicine and Hygiene 68: 479-490.

10. Wong MM and Suter PF (1979). Indirect fluorescent antibody test in occult dirofilariosis. American Journal of Veterinary Research 40: 414-420.

11. Wong MM, Guest MF and Lavoipierre MMJ (1974). Dirofilaria immitis; fate and immunogenicity of irradiated infective stage larvae in beagles. Experimental Parasitology 35: 465-474.

12. Yamagata GR, Gershwin LJ and Wong MM (1992). Immunoglobulin E recognition of Dirofilaria immitis antigens is more specific than immunoglobulin G. Veterinary Parasitology 44: 223-245. 